Journal of Computer Science 4 (12): 967-975, 2008

ISSN 1549-3636

(C) 2008 Science Publications

\title{
A Novel Approach to Modeling and Flooding in Ad-hoc Wireless Networks
}

\author{
Othman Issaad, Samuel Pierre, Gabriel Ioan Ivascu and Oscar Garcia \\ Department of Computer and Software Engineering, Mobile Computing and Networking Laboratory, \\ Station Centre-ville, Montréal (Québec) Canada H3C 3A7
}

\begin{abstract}
This study proposes a new modeling approach for wireless ad-hoc networks. The new approach is based on the construction of fuzzy neighborhoods and essentially consists of assigning a membership or importance degree to each network radio link which reflects the relative quality of this link. This approach is first used to model the flooding problem and then an algorithm is proposed to solve this problem which is of a great importance in ad-hoc wireless networks intrinsically subject to a certain level of node mobility. Simulations carried out in a dynamic environment show promising results and stability compared to the enhanced dominant pruning algorithm. Such an approach is suitable to take into account the volatile aspect of radio links and the physical layer uncertainty when modeling these networks, particularly when the physical layer offers no or insufficient guaranties to high-level protocols as for the flooding.
\end{abstract}

Key words: Ad-hoc wireless networks, auto-configurable networks, flooding, fuzzy sets

\section{INTRODUCTION}

Ad-hoc networks differ from other forms of wireless networks by a total absence of fixed infrastructure. An unspecified number of mobile terminals exploit the radio range of their antennas to create a point to point communication links and form an ad-hoc network ${ }^{[14]}$. In addition to the simplicity of their deployment and exploitation, ad-hoc networks offer users total mobility. Unfortunately, this mobility raises severe problems when establishing radio links. Due to the mobility, the topology of such networks is in perpetual change without notice. Consequently, the radio links become highly volatile, which compromises the guarantee of consistency and presence of these networks. Given these conditions, it is necessary to make extensive discovery operations and/or route and/or neighborhood updates. These operations are carried out through protocols which are known as flooding.

Flooding consists of sending a message from a source to all of the destinations in the network. Typically, this is done as a step by step operation where each mobile unit keeps retransmit ting the same message to its neighbors until the network coverage is completed. This type of mechanism is very frequently used in ad-hoc networks because of random changes of topology. In fact, flooding is particularly useful for route discovering, paging or network broadcasting. In spite of its importance, the physical layer offers no guarantees for delivering a flooding packet. Flooding also consumes much resource and must be used with caution. In fact, using it in a non-effective way, even just a few times, can quickly lead to network overload. By nature, flooding requires a large number of almost simultaneous retransmissions to achieve its functionality and network overloads can occur quickly.

Several solutions have been proposed in the literature in order to reduce the network load induced by flooding. The first wave of broadcasting schemes $^{[1,4,9,10]}$ include the PDP (Partial Dominant Pruning algorithm) ${ }^{[10]}$ which uses a dominating sets approach to reduce the number of nodes that relay the broadcasted packet. The PDP algorithm is based on the utilization of two-hop neighborhood information and a greedy algorithm ${ }^{[7,9]}$ which tries to select broadcasting set of neighbors approaching a minimum-connecteddominating set.

More recent approaches ${ }^{[6,12,20,21]}$ have built on and improved the approaches proposed by the first wave of solutions. For example, Tavli and Henzeilman ${ }^{[21]}$ propose an energy-efficient network-wide voice broadcasting architecture for MANETs organized around overlapping clusters, where the cluster heads create a no connected dominating set through a distributed algorithm. These cluster heads are used to

Corresponding Author: Samuel Pierre, Department of Computer and Software Engineering, Ecole Polytechnique de Montréal, P.O. Box 6079, Station Centre-ville, Montréal (Québec) Canada H3C 3A7

Tel: (514) 340-4711 Ext. 4685 Fax: (514) 340-4658 
efficiently flood the network. Multiple mechanisms are provided to ensure the maintenance of the connected dominating set. Taking on a completely different approach, Agrawal and Zhang ${ }^{[20]}$ propose a probabilistic broadcasting scheme that dynamically adjusts the rebroadcasting probability according to the nodes' distribution and movement. This is realized based on locally available information and without requiring any distance measurements.

In spite of the satisfactory theoretical results of these methods, they do not take into account the physical layer uncertainties which occur when delivering a flooding packet.

This study proposes a new approach for modeling ad-hoc networks which makes it possible to take into account the physical layer uncertainty. This approach is then used to solve the flooding problem.

\section{Background and motivations Definitions}

Fuzzy set: If $X$ is a collection of objects denoted generally by $\mathrm{x}$, then a fuzzy set $\tilde{\mathrm{A}}$ in $\mathrm{X}$ is a set of ordered pairs: $\tilde{\mathrm{A}}=\left\{\left(\mathrm{x}, \mu_{\tilde{\mathrm{A}}}(\mathrm{x})\right) / \mathrm{x} \in \mathrm{X}\right\} \cdot \mu_{\tilde{\mathrm{A}}}(\mathrm{x})$ is called the membership function or grade of membership of $x$ in $\tilde{A}$ that maps $X$ to the membership space $M$. The range of the membership function is a subset of the nonnegative real numbers whose supremum is finite. The membership function is not limited to values between 0 and 1 . If $\sup _{x}\left(\mu_{\tilde{\mathrm{A}}}(\mathrm{x})\right)=1$, the fuzzy set $\tilde{\mathrm{A}}$ is called normal.

Convex fuzzy set: A fuzzy set $\tilde{A}$ is convex if: $\mu_{\tilde{\mathrm{A}}}(\lambda \mathrm{x}+(1-\lambda) \mathrm{y}) \geq \min \left\{\mu_{\tilde{\mathrm{A}}}(\mathrm{x}), \mu_{\tilde{\mathrm{A}}}(\mathrm{y})\right\}, \mathrm{x}, \mathrm{y} \in \mathrm{X}, \lambda \in[0,1]$.

Fuzzy number: A fuzzy number $\tilde{M}$ is a convex normalized fuzzy set of the real line $\mathfrak{R}$ such that:

- $\exists ! \mathrm{x}_{0} \in \mathfrak{R}: \mu_{\tilde{\mathrm{M}}}\left(\mathrm{x}_{0}\right)=1\left(\mathrm{x}_{0}\right.$ is called the mean value of M )

- $\mu_{\tilde{\mathrm{M}}}(\mathrm{x})$ is piecewise continuous

$\begin{array}{cll}\text { An example of a fuzzy } & \text { number is } \\ \tilde{M}=\left\{\left(x, \mu_{\tilde{\mathrm{A}}}(\mathrm{x})\right) / \mu_{\tilde{\mathrm{A}}}(\mathrm{x})=1 /\left(1+(\mathrm{x}-5)^{2}\right)\right\} . & \tilde{\mathrm{M}} \text { is with }\end{array}$ mean $\mathrm{x}_{0}=5$ and could be called approximately 5 . It is represented as shown in Fig. 1.

Fuzzy sets are usually considered to have real (crisp) membership function but they are not restricted to this condition. Fuzzy sets could be extended to ones

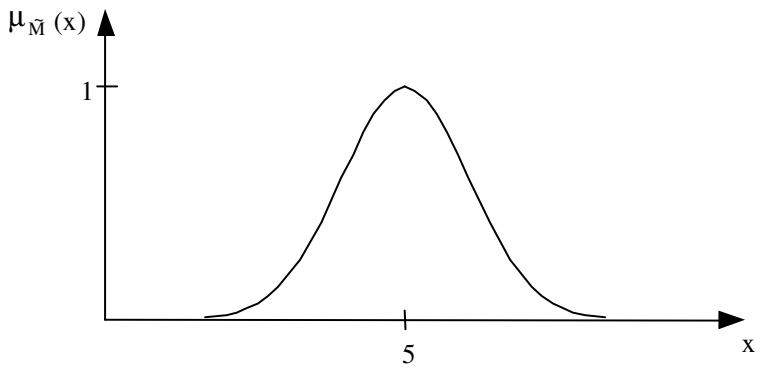

Fig. 1: Fuzzy number approximately 5

whose membership function itself is a fuzzy set. If we call fuzzy sets, such as those considered so far, type 1 fuzzy sets, then a type 2 fuzzy set can be defined as follows:

Type 2 fuzzy set: A type 2 fuzzy set is a fuzzy set whose membership values are type 1 fuzzy sets on $[0,1]$.

Motivation: An ad-hoc network is generally modeled by a graph $\mathrm{G}(\mathrm{N}, \mathrm{A})$ where $\mathrm{N}$ denotes the set of nodes representing the mobile units and $\mathrm{A}$ the set of arcs representing the established radio links. Modeling adhoc networks and the existence of these networks are based on the concept of neighborhoods.

Each network node u could define a subset $\mathrm{V}(\mathrm{u}) \subseteq$ $\mathrm{N}$ of its neighbors, that is, a subset composed of all the nodes $\mathrm{v}$ of $\mathrm{N}$ which receive a minimal power threshold of u's radio signal. When this power threshold is exceeded, the radio link between both mobile units $u$ and $\mathrm{v}$ is established, which leads to an arc binding the two mobiles in the graphical representation of the adhoc network.

A couple of mobile nodes $u$ and $v$ could be in one of the following situations: Either there is an $\operatorname{arc}(\mathrm{u}, \mathrm{v})$ binding them, which means that $v$ receives u's signal $(\mathrm{v} \in \mathrm{V}(\mathrm{u}))$ or there is no such arc, which means that $\mathrm{v}$ does not receive u's signal. Due to various factors including the mobility, an ad-hoc unit does not lose a signal in such a discrete manner (Fig. 2a) but this signal passes through different levels going from good, to average, to null. To encounter this modeling problem, a real weight is usually associated to each arc in order to quantify the quality of the radio link that it represents. This leads to the continuous curve (Fig. 2b) which reasonably approaches the real phenomenon of radio signal degradation.

This phenomenon is complex and introduces random fluctuations which make it quite difficult to evaluate precisely the quality of a radio link ${ }^{[17]}$. Moreover, at near critical power values, the existence of 


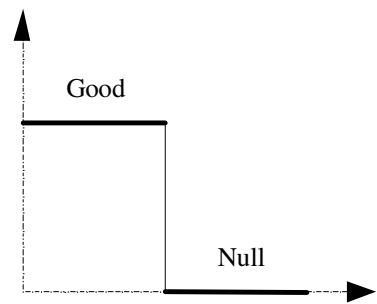

(a) Two-state approach

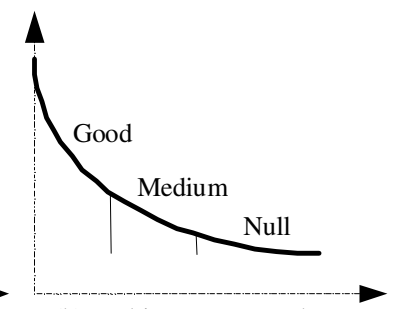

(b) Multi-state approach
Fig. 2: Approximation of signal degradation

radio links is even more uncertain. It becomes impossible to associate an adequate real weight to each arc.

In the classical approach, the existence of a radio link can only be revoked during time. This compromises the volatile aspect of these networks and radio links.

There is evidently a need to find better and more realistic ways to model the connectivity that exists between nodes within ad-hoc wireless networks. One promising approach, presented hereafter, is based on fuzzy sets and set memberships. In our approach, all radio links connecting nodes within a given neighborhood are assigned a certain membership degree. Unlike the classical approach, this allows to model radio link uncertainty and take into account the volatile nature of ad-hoc wireless networks.

A novel approach to modeling ad-hoc wireless networks: In this study, a new approach is proposed for modeling wireless ad-hoc networks which is better adapted to take into account the specificities of such networks. Then, this approach is used to model and solve the flooding problem.

Basic principles: The new approach that we propose for modeling ad-hoc networks is based on the construction of type 2 fuzzy neighborhoods. Indeed, instead of considering whether or not mobile unit $v$ belongs to $\mathrm{V}(\mathrm{u})$, the neighborhood of an other mobile $\mathrm{u}$, we consider that $\mathrm{v}$ belongs to $\mathrm{V}(\mathrm{u})$ with a certain membership degree that reflects the quality of the radio link (u,v). Then, instead of considering ad-hoc network as a set of terminals, each defining a neighborhood using a logical membership (true or false), we build a neighborhood for each mobile unit using a fuzzy valued membership function. This will allow to model in a completely simple and natural way the volatile aspect and the physical layer uncertainty inherent to ad-hoc networks.

According to the proposed approach, we model an ad-hoc network by a fuzzy graph $\tilde{\mathrm{G}}(\tilde{\mathrm{N}}, \tilde{\mathrm{A}})$ that could be oriented or not, depending on the nature of the radio links. $\tilde{\mathrm{N}}$ is a type 2 fuzzy set on $\mathrm{N}$ and $\tilde{\mathrm{A}}$ is a fuzzy relation on $\mathrm{N} \times \mathrm{N} \rightarrow \tilde{\mathfrak{R}}$ where $\tilde{\mathfrak{R}}$ is the set of fuzzy numbers. In such a graph, each arc $(\mathrm{u}, \mathrm{v})$ is assigned a membership degree $\mu(\mathrm{u}, \mathrm{v})$ which reflects the quality of the radio link $(\mathrm{u}, \mathrm{v})$. We also assign a membership degree to each node if mobile units have different roles or importance in the network. This importance can represent, for example, the availability of each node to perform certain network or protocol tasks regarding its local properties such as load or throughput. Furthermore, by letting all the membership functions to take fuzzy values, we model the volatile aspect of radio links and the physical layer uncertainty, especially when this layer offers no or insufficient guarantees to high-level protocols.

Problem modeling: Let $\mathrm{N}$ denotes the set of all nodes forming an ad-hoc network and A the set of all possible arcs or radio links which could be established during the network life. As mentioned above, we represent an ad-hoc network by a fuzzy graph $\tilde{\mathrm{G}}(\tilde{\mathrm{N}}, \tilde{\mathrm{A}})$ where $\tilde{\mathrm{N}}$ is a (type 2) fuzzy set on $N$ and $\tilde{A}$ is a fuzzy relation on $\mathrm{N} \times \mathrm{N}$ which verifies:

$$
\forall(\mathrm{u}, \mathrm{v}) \in \mathrm{N} \times \mathrm{N} \mu_{\tilde{\mathrm{A}}}(\mathrm{u}, \mathrm{v}) \leq \min \left(\mathrm{u}_{\tilde{\mathrm{N}}}(\mathrm{u}), \mu_{\tilde{\mathrm{N}}}(\mathrm{v})\right)
$$

where $\mu$ denotes the membership function. Nevertheless, in the flooding context, we could suppose that: $\forall \mathrm{u} \in \tilde{\mathrm{N}} \quad \mu_{\tilde{\mathrm{N}}}(\mathrm{u})=1$. This means that we consider that all network nodes have the same importance in a flooding operation. In other words, we consider that each mobile can start or participate in any flooding operation that would be running at a given time. Under this condition, we obtain $\tilde{\mathrm{N}}=\mathrm{N}$ and the inequality (1) can then be deleted.

Modeling an ad-hoc network by a fuzzy graph is achieved by constructing around each mobile node $u \in \tilde{N}$ a fuzzy set of neighbors $\tilde{V}(u)$ where each node in the neighborhood is assigned a membership degree to that set. This can be accomplished by setting the following conditions:

$$
\forall(\mathrm{u}, \mathrm{v}) \in \mathrm{N} \times \mathrm{N}, \mathrm{u}_{\tilde{\mathrm{v}}(\mathrm{u})}(\mathrm{v})=\mu_{\tilde{\mathrm{A}}}(\mathrm{u}, \mathrm{v}) \text { and } \mu_{\tilde{\mathrm{v}}(\mathrm{u})}(\mathrm{u})=1
$$

The membership degree reflects the quality of the radio link $(\mathrm{u}, \mathrm{v})$ or the power received at $\mathrm{v}$.

Let us consider two mobile units, $u$ and $v$, presumably provided with omni-directional antenna of 
gains $\mathrm{G}_{\mathrm{u}}$ and $\mathrm{G}_{\mathrm{v}}$, running at a frequency $f$ with a broadcast power $\mathrm{P}_{\mathrm{u}}$ and $\mathrm{P}_{\mathrm{v}}$ respectively. Let $\rho$ denote the distance between $\mathrm{u}$ and $\mathrm{v}$. The received power at $v$ can be expressed as ${ }^{[15]}$.

$$
P_{u}(v)_{[d \mathrm{~B}]}=10 \log _{10} \cdot\left(G_{v}\left(\frac{c}{4 \pi f}\right)^{2} G_{u} P_{u}\right)-\alpha \cdot 10 \log (\rho)+X_{\sigma}
$$

where c represents the light speed, $\alpha(2 \leq \alpha \leq 6)$ is called the path-loss exponent and $\mathrm{X}_{\sigma}$ is a zero-mean Gaussian random variable (in $\mathrm{dB}$ ) with standard deviation $\sigma$ also in $\mathrm{dB}$. Let us define:

$$
h(\rho, \alpha)=10 \cdot \log _{10}\left(G_{v} \cdot\left(\frac{c}{4 \pi f}\right)^{2} \cdot G_{u} P_{u}\right)-\alpha \cdot 10 \log (\rho)
$$

Then, $\mathrm{P}_{\mathrm{u}}(\mathrm{v})_{[\mathrm{dB}]}=\mathrm{h}(\rho, \alpha)+\mathrm{X}_{\sigma}$.

$\mathrm{X}_{\sigma}$ is a zero-mean Gaussian random variable, hence its probability density function is continuous and convex. $\mathrm{X}_{\sigma}$ could be used to construct what we call the fuzzy received power $\tilde{\mathrm{P}}_{\mathrm{u}, \sigma}(\mathrm{v})$ as a fuzzy number $\tilde{\mathrm{P}}_{\mathrm{u}, \sigma}(\mathrm{v})=\left\{\left(\mathrm{x}, \mu_{\tilde{\mathrm{P}}}(\mathrm{x})\right) / \mu_{\tilde{\mathrm{P}}}(\mathrm{x})=\mathrm{e}^{-\frac{(\mathrm{x}-\mathrm{h}(\rho, \alpha))^{2}}{2 \sigma^{2}}}\right\}$. This return to construct around the real-mean-power value $h(\rho, \alpha)$ a fuzzy set $\tilde{\mathrm{P}}_{\mathrm{u}, \sigma}(\mathrm{v})$ whose membership function $\mu_{\tilde{\mathrm{P}}}$ is illustrated in Fig. 3. Actually, the membership degree of each real power value $h(\rho, \alpha)+X_{\sigma}$ reflects the probability of this value according to the probability density function which characterizes $\sigma$ corrected to the factor of $\sigma \sqrt{2 \pi}$. Finally:

$$
\mu_{\tilde{v}(\mathrm{u})}(\mathrm{v})=\frac{1}{\sigma \sqrt{2 \pi}} \tilde{\mathrm{P}}_{\mathrm{u}, \sigma}(\mathrm{v})
$$

Nevertheless, $\mu_{\tilde{\mathrm{P}}}$ depends on the three unknown parameters: $\rho, \alpha$ and $\sigma$. Those are essentially distance,

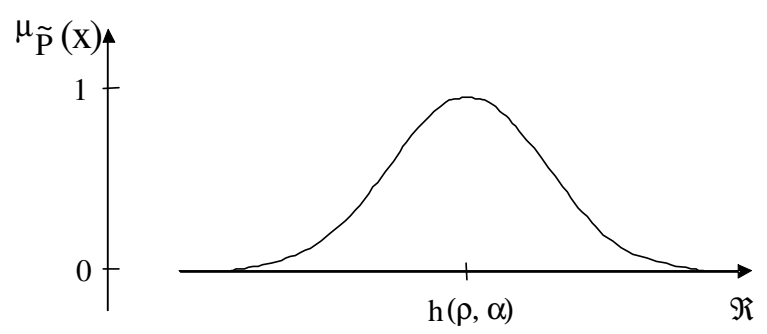

Fig. 3: Definition of the fuzzy power $\tilde{\mathrm{P}}$ environment and frequency dependent and should be estimated via site measurements ${ }^{[15]}$. On the other hand, the transmitter/receiver distance $\rho$ changes and must be calculated every time a broadcasting mobile u needs to establish its fuzzy neighborhood. This can be achieved by using an estimation of the average received power ${ }^{[11]}$ when $u$ receives a message from each of its neighbors. In fact, mobile ad-hoc units must send hello messages periodically to notify their presence. Using that packet or any other packet that $\mathrm{u}$ would have received, it can estimate the distance of each of its neighbors $v$ by:

$$
\rho=\left(G_{u} \cdot\left(\frac{c}{4 \pi f}\right)^{2} \cdot \frac{G_{v} P_{v}}{P_{v}(u)}\right)^{1 / \alpha}
$$

Solving strategy and algorithm: The concept of neighborhood is very important when optimizing the flooding process. The optimum can be reached when each node has complete neighborhood information and knows the complete topology of the network. However, collecting such information over a large number of hops and keeping it updated consumes much resource and can rapidly overload the network. Thus, we limit the collection of topology information to two-hops as this assumption was tested and yielded satisfactory results $^{[9]}$.

Henceforth, let us consider that each node is aware of its neighborhood information over two-hops. Since the flooding is iterative, we will treat at each iteration a restricted two-hop network around the broadcasting node.

Suppose that a node $\mathrm{u}$ received a flooding packet from a node $\mathrm{m}$ and should relay it to complete the flooding. In a two-hops strategy, u will select some of its adjacent nodes $(\in \mathrm{V}(\mathrm{u}))$ which should relay the packet to cover $\mathrm{U}=\mathrm{V}(\mathrm{V}(\mathrm{u}))^{[9]}$. However, it is not necessary to cover all $\mathrm{V}(\mathrm{V}(\mathrm{u}))$ nodes. In fact, some of these have already received the packet, for instance, when $\mathrm{m}$ sent it. Actually, $\mathrm{U}$ could be restricted to $\mathrm{U}=\mathrm{V}(\mathrm{V}(\mathrm{u}))-\mathrm{V}(\mathrm{u})-\mathrm{V}(\mathrm{m})-\mathrm{V}(\mathrm{V}(\mathrm{u}) \cap \mathrm{V}(\mathrm{m}))^{[10]}$.

Then the problem to solve is the following: Select, in the neighborhood $\mathrm{V}(\mathrm{u})$, a minimum subset of nodes that should relay the flooding packet to cover all $\mathrm{U}$ nodes. Taking into account our modeling strategy, the problem becomes: Select, in the fuzzy neighborhood $\tilde{\mathrm{V}}(\mathrm{u})$, a minimum subset of nodes that should relay the flooding packet to cover all $U$ nodes. If $\mu_{\tilde{\mathrm{v}}(\mathrm{x})}(\mathrm{y})=1, \forall \mathrm{x}, \mathrm{y} \in \mathrm{V}(\mathrm{u}) \cup \mathrm{U}$, the problem turns to a connected dominating set which is NP-hard ${ }^{[9]}$.

To solve this problem, we will split it into two subproblems. The first addresses the users' mobility and its 


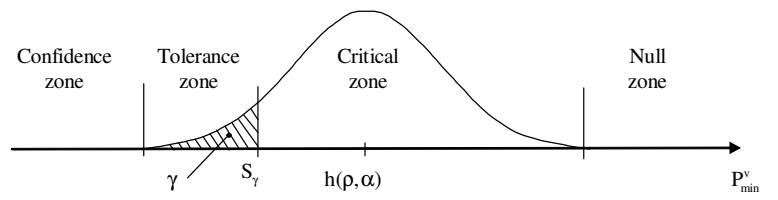

Fig. 4: Definition of the loss-tolerance-threshold $\mathrm{S}_{\gamma}$

effects on the quality of the radio links. We will try to limit the number of failed broadcasts due to topology changes and radio link failure. So, we determine a subset of nodes known as critical and use the best available radio links to deliver them the flooding packet. In fact, a node $w \in U$ is said to be critical if the best two-hops available path ( $\mathrm{u}, \mathrm{v}, \mathrm{w})$ contains a weak radio link. A $(\mathrm{u}, \mathrm{v})$ radio link is considered to be weak if the minimal threshold power $\mathrm{P}_{\min }^{\mathrm{v}}$ that $v$ is able to receive falls to exceed the threshold $S_{\gamma}$ shown in Fig. 4. $\mathrm{S}_{\gamma}$ is defined in relation to the fuzzy received power at $\mathrm{v}$ by giving a value to $\gamma$ which determines the tolerance to flooding-packet-loss and can be adjusted depending on the context.

By definition, the flooding packet will probably not reach the critical nodes in spite of using the best routes. Then, we will attempt to deliver them the flooding packet several times. For that purpose, we redefine the set of nodes to cover, as the following: $\mathrm{T}=\mathrm{U} \cup \mathrm{S}$, where $\mathrm{S}$ is the set of critical nodes of $\mathrm{m}$ which belong to $\mathrm{V}$ $(\mathrm{V}(\mathrm{u}))$. The first sub-problem could be solved using a Fuzzy Shortest Path (FSP) algorithm ${ }^{[3,17,18]}$. For our particular approach, we have chosen to use the generalized path-finding algorithm developed by Boulmakoul ${ }^{[3]}$ for finding the FSPs in the fuzzy graph.

The second sub-problem consists of reducing the number of necessary broadcasts to cover non-critical nodes. This can be achieved by using an approximation algorithm for Minimum-Connected-Dominating Sets $(\mathrm{MCDS})^{[7,9,10]}$. To find the connected dominating set in the second sub-problem, we have used the greedy algorithm proposed by Guha ${ }^{[7]}$.

Let $\tilde{\mathrm{G}}(\tilde{\mathrm{N}}, \tilde{\mathrm{A}})$ be an oriented fuzzy graph representing the two-hop neighborhood of $u$. Let $\mu_{\tilde{v}(x)}(y)=0$, for each $x, y \in V(u) \backslash\{u\} \cup T$. Let us define for each $\operatorname{arc}(\mathrm{x}, \mathrm{y})$ a fuzzy weight $\tilde{\mathrm{p}}$ as follows: $\tilde{\mathrm{p}}(\mathrm{x}, \mathrm{y})=\mu_{\tilde{\mathrm{v}}(\mathrm{x})}(\mathrm{y})$. Under these assumptions and a chosen value for $\gamma$, the $\gamma^{+}$algorithm that we propose is shown in Fig. 5.

The algorithm works as follows: Let us consider the two-hops neighborhood shown in Fig. 6. In this case,

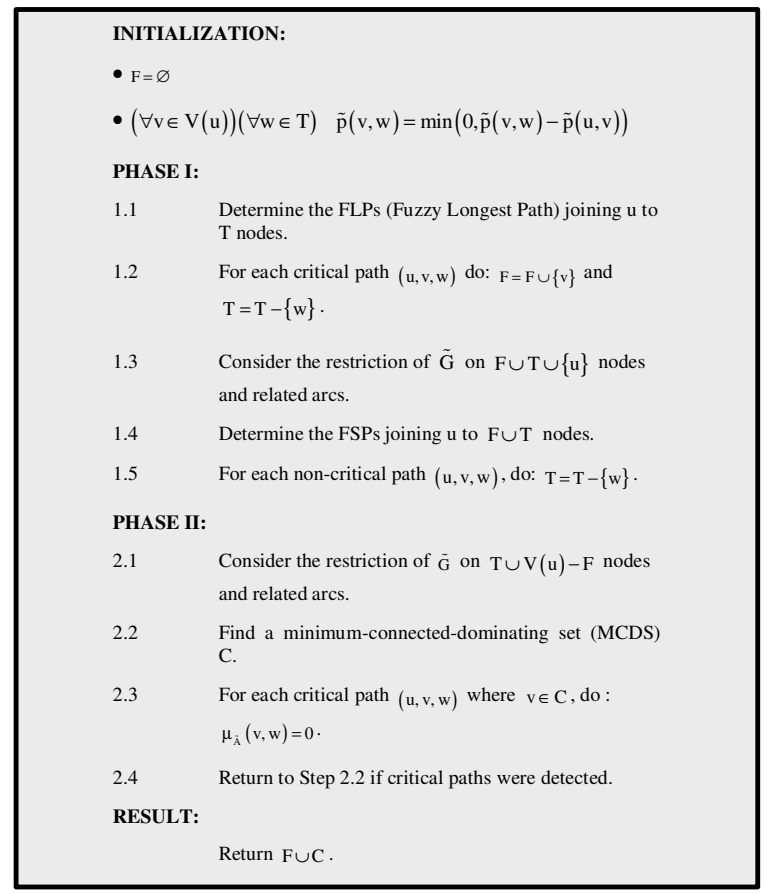

Fig. 5: $\gamma+$ flooding algorithm

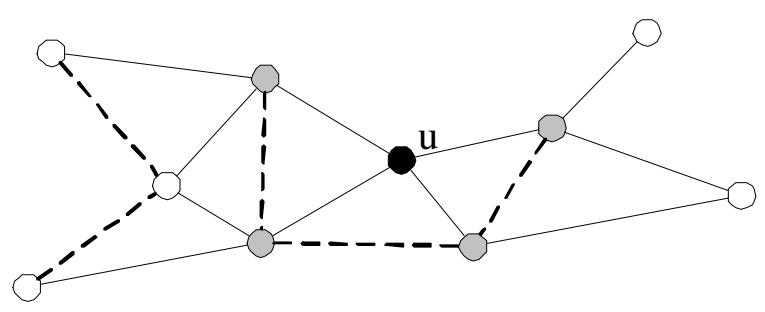

Fig. 6: Definition of V(u) and T

$\mathrm{V}(\mathrm{u})$ is composed of all gray nodes whereas $T$ is composed of white nodes. Then the algorithm will use the gray nodes to deliver the flooding message to the white ones. Prior to using the algorithm, we eliminate all intra $\mathrm{V}(\mathrm{u}) \backslash\{\mathrm{u}\}$ arcs as well as intra $\mathrm{T}$ arcs represented by the dashed arcs in Fig. $6\left(\mu_{\tilde{v}(x)}(y)=0\right.$, for each $\mathrm{x}, \mathrm{y} \in \mathrm{V}(\mathrm{u}) \backslash\{\mathrm{u}\}$ or $\mathrm{x}, \mathrm{y} \in \mathrm{T})$. These arcs will not be used in a two-hops strategy.

The first step of the algorithm is the initialization step. For now, the first phase forwarding nodes set is empty $(\mathrm{F}=\varnothing)$ and we adapt the arcs' weights $(\forall \mathrm{v} \in \mathrm{V}(\mathrm{u}))(\forall \mathrm{w} \in \mathrm{T}) \quad \tilde{\mathrm{p}}(\mathrm{v}, \mathrm{w})=\min (0, \tilde{\mathrm{p}}(\mathrm{v}, \mathrm{w})-\tilde{\mathrm{p}}(\mathrm{u}, \mathrm{v}))$ to have the length of each two-hops path $(\mathrm{u}, \mathrm{v}, \mathrm{w})$ equals $\min (\tilde{\mathrm{p}}(\mathrm{u}, \mathrm{v}), \tilde{\mathrm{p}}(\mathrm{v}, \mathrm{w}))$. In fact, Length $(\mathrm{u}, \mathrm{v}, \mathrm{w})=\tilde{\mathrm{p}}(\mathrm{u}, \mathrm{v})+\tilde{\mathrm{p}}(\mathrm{v}, \mathrm{w})$ then Length $(\mathrm{u}, \mathrm{v}, \mathrm{w})=$ $\tilde{\mathrm{p}}(\mathrm{u}, \mathrm{v})+\min (0, \tilde{\mathrm{p}}(\mathrm{v}, \mathrm{w})-\tilde{\mathrm{p}}(\mathrm{u}, \mathrm{v}))=\min (\tilde{\mathrm{p}}(\mathrm{u}, \mathrm{v}), \tilde{\mathrm{p}}(\mathrm{v}, \mathrm{w}))$. 
The first phase of the algorithm starts. In step 1.1, we calculate the fuzzy longest paths $(\mathrm{u}, \mathrm{v}, \mathrm{w})$ from $\mathrm{u}$ to cover all $\mathrm{T}$ nodes, these paths are the strongest ones to cover the w nodes. In step 1.2, we identify among these paths, all the critical ones. Even though these paths are critical to cover the $\mathrm{w}$ nodes, they must be used since they are the best ones found. So we hold $\mathrm{v}$ as forwarding nodes and $\mathrm{w}$ as possibly covered nodes: $\mathrm{F}=\mathrm{F} \cup\{\mathrm{v}\}$ and $\mathrm{T}=\mathrm{T}-\{\mathrm{w}\}$. Now $\mathrm{F}$ contains some forwarding $\mathrm{V}$ nodes and the set of $\mathrm{T}$ nodes to cover is smaller. Since all $\mathrm{V}$ nodes belonging to $\mathrm{F}$ will be forwarding the flooding message, we'll try to find out if we can use the forwarded $\mathrm{v}$ messages to cover other $\mathrm{T}$ nodes and makes it become smaller. So in step 1.3 we consider the restriction of $\tilde{G}$ on $F \cup T \cup\{u\}$. If the weakest paths $(\mathrm{u}, \mathrm{v}, \mathrm{w})$ going through the forwarding nodes $\mathrm{v}$ and reaching some $\mathrm{T}$ nodes $\mathrm{w}$ are not critical, then these paths could definitely be used to cover the reached $\mathrm{T}$ nodes. In this case, the $\mathrm{w}$ nodes could be considered as covered and removed from $\mathrm{T}$ : $\mathrm{T}=\mathrm{T}-\{\mathrm{w}\}$.

The second phase of the algorithm starts by considering the restriction of $\tilde{G}$ on the remaining nodes $\mathrm{T} \cup \mathrm{V}(\mathrm{u})-\mathrm{F}$. For all remaining $\mathrm{T}$ nodes we are sure to find at least one path $(\mathrm{u}, \mathrm{v}, \mathrm{w})$ which is not critical to cover them, otherwise they would have been eliminated by the first phase of the algorithm. In step 2.2, we find a minimum-connected-dominating set $C$. This set contains all forwarding nodes $v$ needed to cover all remaining $\mathrm{T}$ nodes. If a critical path $(\mathrm{u}, \mathrm{v}, \mathrm{w})$ is found, we eliminate it in step 2.3 and restart the process of building $\mathrm{C}$. The second phase of the algorithm ends by finding a minimum-connected-dominating set $C$ which does not contain any critical path and cover all remaining $\mathrm{T}$ nodes.

The algorithm ends by returning the set of all forwarding nodes: FuC

Algorithm termination criterion: Under certain circumstances, performing the flooding using a two-hop strategy can lead to an infinite loop of retransmission requests even if all network nodes have received the flooding packet. In these conditions, a termination criterion is absolutely necessary.

An effective termination criterion proposed in ${ }^{[10]}$ is that a node is not allowed to retransmit a flooding packet which it has already transmitted. Even if this termination criterion breaks out the infinite loop, it still generates unnecessary broadcasts. In effect, suppose that a mobile node $\mathrm{u}$ receives for the first time a flooding packet $\mathrm{P}$. If $\mathrm{u}$ 's $\mathrm{Id}$ is piggybacked to $\mathrm{P}$, then $\mathrm{u}$ should broadcast the packet and this broadcast is necessary to cover u's neighborhood. However, if u's Id is not included in $\mathrm{P}$, then $\mathrm{u}$ should not relay the packet and its transmission is unnecessary to cover its neighborhood. In both cases, if $\mathrm{u}$ receives another copy of $\mathrm{P}$ with its Id included, it should not retransmit it since its neighborhood has already been covered. Thus, an improved termination criterion would be that a node is not allowed to retransmit a flooding packet that it has previously received. This is the criterion that was chosen for the $\gamma+$ algorithm.

\section{RESULTS}

We measured the performance of $\gamma+$, PDP and basic broadcast flood algorithms using computer simulations. The algorithms were implemented in QualNet Simulator 3.9, a scalable packet-level simulator with accurate radio and mobility models. The simulator implements various routing, MAC protocols as well as physical layer functions.

We were interested in determining three performance indices: the average number of covered nodes, the average number of forward nodes and the average number of failed transmissions. However, in order to compare the two algorithms and to better assess their performance, we standardized these indices. Then, we calculated the percentage of coverage (\%Cover), the density of transmissions (\% Tx) and the density of failed transmissions (\%TxFail) as follows:

$$
\begin{aligned}
& \text { \%Cover }=\frac{\text { NbCoveredNodes }}{\text { NbNodes }} \cdot 100 \\
& \% \mathrm{Tx}=\frac{\text { NbForwardNodes }}{\text { NbCoveredNodes }} \cdot 100 \\
& \% \text { TxFail }=\frac{\text { NbFailedTransmissions }}{\text { NbCoveredNodes }} \cdot 100
\end{aligned}
$$

The simulator randomly generates an ad-hoc network consisting of NbMobile $\in\{20,30,40,50,60$, $70,80,90,100\}$ randomly scattered in a square broadcasting area of $1,500 \times 1,500 \mathrm{~m}$. Each mobile is provided with an omni-directional antenna and running at a variable speed registered in $\left[\mathrm{V}_{\min }, \mathrm{V}_{\max }\right] \in\{[0,0]$, $\left.[0,5],[5,10],[10,15],[15,20] \mathrm{m} \mathrm{sec}^{-1}\right\}$. For each scenario consisting of a random combination (NbMobile, $\mathrm{V}_{\min }, \quad \mathrm{V}_{\max }$ ), 50 experiments were conducted. All experiments were simulated under 


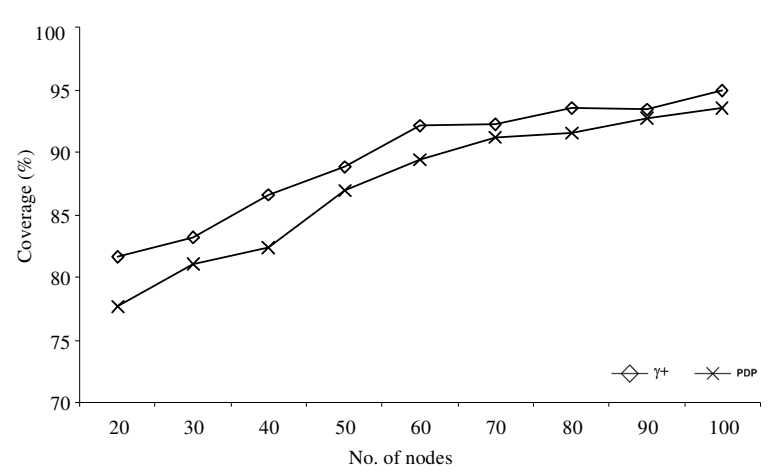

Fig. 7: Coverage percentage as a function of the number of nodes for $\gamma+$ and PDP

realistic channel conditions including multipath fading, constant shadowing and two-ray propagation model as provided by the QualNet simulator.

Coverage percentage: Figure 7 shows the coverage percentage performance as a function of the network's node density for $\gamma+$ and PDP. We can see that the performance of both algorithms improves as the network density increases (i.e., the number of nodes increases). However, we can notice that $\gamma+$ performance exceeds that of PDP regardless of the network's density. Indeed, on the whole density interval, $\gamma+$ provides a broadcast coverage over $90 \%$, on average, while PDP's coverage over the same interval remains just under $87 \%$, on average. More particularly, in the $[20,60]$ nodes interval, $\gamma+$ improves PDP's coverage by slightly more than $3 \%$, on average. On the other hand, $\gamma+$ advantage over PDP decreases as the network density increases. Even though the coverage difference between the algorithms is not significant, $\gamma+$ reduces the network load significantly compared to PDP when the network density increases, as will be shown later.

Figure 8 shows that $\gamma+$ offers better performance than PDP when high-level coverage is needed. Indeed, $\gamma+$ yields a coverage inside the $[80,100]$ interval around $92 \%$ of the times whereas PDP yields an $85 \%$ frequency for the same interval. This shows that $\gamma+$ is algorithmically more stable than PDP as its coverage percentage's standard deviation is $8.77 \%$ compared to $12.30 \%$ for PDP.

Network load: The network load (\%Tx) performance as a function of the network size for both algorithms is shown in Fig. 9. Again, we notice that $\gamma+$ offers better performances than PDP over the [20,100] node density interval. As a matter of fact, over the whole interval $\gamma+$ yields a 52.29\% network load, on average while PDP yields a network load slightly under $60 \%$, implying an

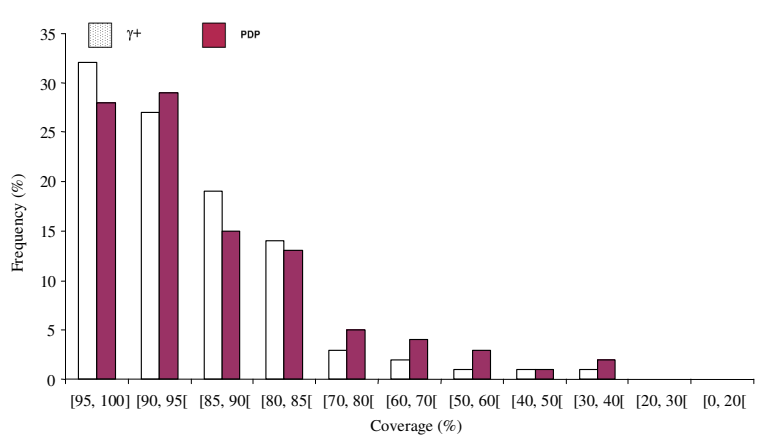

Fig. 8: Coverage percentage frequency for PDP and $\gamma+$

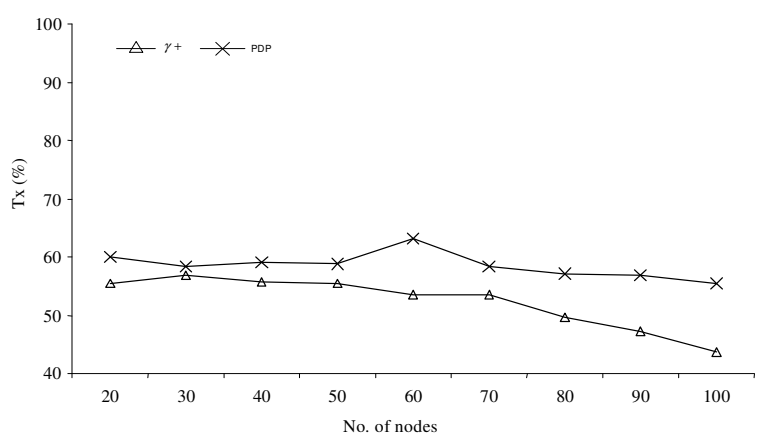

Fig. 9: Network load (\%Tx) as a function of the number of nodes for PDP and $\gamma+$

$8 \%$ decrease of the number of forwarding nodes for $\gamma+$. Recall that the network load, as defined earlier, represents the forwarding nodes density and is computed as the number of forwarding nodes over the number of covered nodes.

The results obtained by $\gamma+$ were to be expected since one of the design goals of the algorithm was to reduce the number of broadcast message transmissions required to flood the network. We can further observe on the figure that as the network density increases the network load for $\gamma+$ decreases to reach a minimum of $43.5 \%$ for 100 nodes. Moreover, the biggest differences between both algorithm's network load occurs in the $[60,100]$ high density interval. This strengthens the assumption that $\gamma+$ is especially well suited for dense ad hoc networks.

Transmission failures: Figure 10 shows the transmission failure percentage as a function of the number of nodes in the network. At this time, we have already seen that $\gamma+$ improves the coverage percentage and reduces the network overload compared to PDP. In addition to that, Fig. 10 shows that $\gamma+$ also reduces the number of failed transmissions. Indeed, the number of failed transmission for $\gamma+$ is lower than for PDP since 


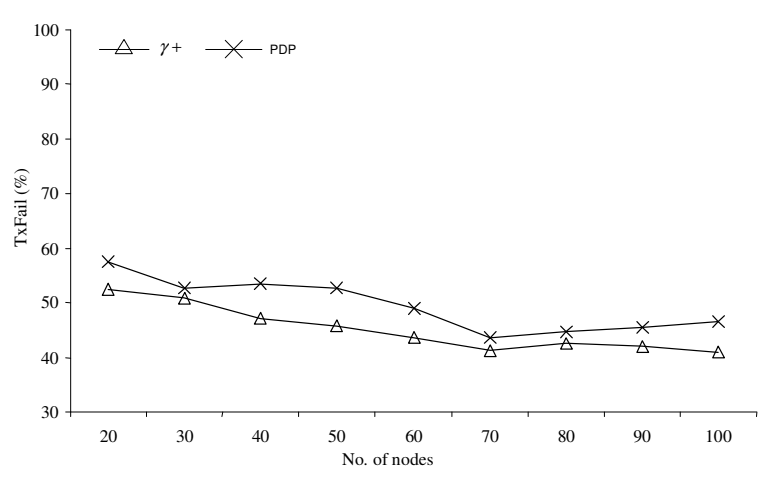

Fig. 10: Failed transmission's density (\%TxFail) as a function of the number of nodes for PDP and $\gamma+$

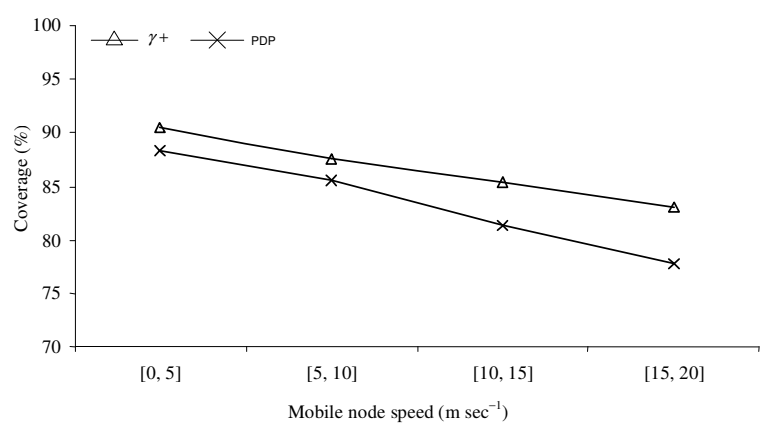

Fig. 11: Coverage percentage as a function of mobile node speed for a 60 nodes network for PDP and $\gamma+$

the number of forwarding nodes and hence the number of broadcast transmissions is lower. Over the whole interval, $\gamma+$ yields a failed transmission rate around $45 \%$ while PDP yields a rate of more than $50 \%$. The greatest differences in performance between the two algorithms belong to the $[40,60]$ and $[90,100]$ intervals where $\gamma+$ improves PDP by 6.27 and $4.45 \%$, respectively.

Coverage percentage with mobile nodes: Finally, we evaluate the performance of both algorithms according to node mobility. As was mentioned earlier, $\gamma+$ 's fuzzy modeling approach takes into account a node's mobility impact on the quality of the links that connect it to its neighbors. By assigning a fuzzy number to a link through a custom set membership function, $\gamma+$ is able to cope in a gentle fashion with the signal degradation caused by a node roaming away from its original position. This characteristic is exemplified in Fig. 11 where $\gamma+$ clearly outshines PDP when mobility is introduced. We have simulated a random waypoint mobility model with a variable speed range. Indeed, over the whole mobility range, $\gamma+$ offers network coverage of over $87 \%$ while PDP's coverage over the same range is just fewer than $83 \%$. The greatest difference between the two algorithms occurs in the $[15,20] \mathrm{m} \mathrm{sec}^{-1}$ speed interval where the coverage offered by $\gamma+$ is $7 \%$ greater than that of PDP.

\section{CONCLUSION}

This study addresses wireless ad-hoc modeling and flooding problems. First of all, we pointed out the limitations of the classical modeling approach and proposed a new one based on the construction of fuzzy sets. This new approach is suitable for wireless ad-hoc networks modeling since it allows to take into account the physical layer uncertainty and the volatile aspect of the radio links. The proposed approach was used to model and solve the flooding problem.

We proposed a two-phase algorithm called $\gamma+$ based on the calculation of fuzzy shortest paths and the approximation of minimum-connected-dominating sets. Simulation results have shown very good performance for the $\gamma+$ algorithm comparing to the PDP and a basic broadcast flood algorithm. In fact, $\gamma+$ improves the network coverage and offers better stability while reducing network load and the number of failed transmissions. We globally noticed the superior performance of $\gamma+$ comparing to PDP regardless of the network density or the mobile speeds. As a result, $\gamma+$ is more suitable for flooding in wireless ad-hoc networks.

\section{REFERENCES}

1. Alzoubi, K.M., P.J. Wan and O. Frieder, 2002. New distributed algorithm for connected dominating set in wireless ad hoc networks. In: Proceedings of the 35th Annual Hawaii International Conference on System Sciences (HICSS'02, Jan. 7-10, IEEE Computer Society Washington, DC, USA., pp: 297.

2. Andersen, J.B., T.S. Rappaport and S. Yoshida, 1995. Propagation measurements and models for wireless communications channels. Commun. Magazine, IEEE., 33: 42-49.

3. Boulmakoul, A., 2004. Generalized path-finding algorithms on semirings and the fuzzy shortest path problem. J. Comput. Applied Math., 162: 263-272.

4. Calinescu, G., I. Mandoiu, P.J. Wan and A. Zelikovsky, 2001. Selecting forwarding neighbors in wireless ad hoc networks. In: Proceeding ACM International Workshop Discrete Algorithms and Methods for Mobile Computing (DIALM '01), Dec. 2001, pp: 34-43. 
5. Cox, D., R. Murray and A. Norris, 1984. $800 \mathrm{Mhz}$ attenuation measured in and around suburban houses. AT and T Bell Laboratories Tech. J., 673: 921-954.

6. Sheng, M., J. Li and Y. Shi, 2005. Relative degree adaptive flooding broadcast algorithm for ad hoc networks. IEEE Trans. Broadcast., 51: 216-222.

7. Guha, S. and S. Khuller, 1998. Approximation algorithms for connected dominating sets. Algorithmica, 20: 374-387.

8. Liberti, J.C. and T.S. Rappaport, 1992. Statistics of shadowing in indoor radio channels at 900 and 1900 MHz. In: Military Communications Conference, 1992. MILCOM '92, Conference Record. 'Communications-Fusing Command, Control and Intelligence', IEEE, Oct. 11-14, 3: 1066-1070.

9. Lim, H. and C. Kim, 2001. Flooding in wireless ad hoc networks. Comput. Commun., 24: 353-363.

10. Lou, W. and $\mathrm{J}$. $\mathrm{Wu}, 2002$. On reducing broadcast redundancy in ad hoc wireless networks. IEEE Trans. Mobile Comput., 1: 111-122.

11. Narasimhan, R. and D.C. Cox, 2001. Estimation of mobile speed and average received power in wireless systems using best basis methods. IEEE Trans. Commun., 49: 2172-2183.

12. Hsu, C.S, Y.T. Tseng and J.P. Sheu, 2007. An efficient reliable broadcasting protocol for wireless mobile ad hoc networks. Ad Hoc Networks, 5: 299-312. Doi: 10.1016/J.Adhoc.2005.11.006

13. Perkins, C., E.M. Royer and I.D. Chakeres, 2004. Ad-hoc on-demand distance vector routing. Mobile Ad Hoc Networking Working Group-Internet Draft, January 2004.
14. Santi, P., 2005. The critical transmitting range for connectivity in mobile ad hoc networks. IEEE Trans. Commun, 4: 310-317.

15. Rappaport, T.S., 2001. Wireless Communications: Principles and Practice. 2nd Edn., Prentice Hall.

16. Seidel, S.Y., T.S. Rappaport, S. Jain, M.L. Lord, R. Singh, 1991. Path loss, scattering and multipath delay statistics in four European cities for digital cellular and microcellular radiotelephone. IEEE Trans. Vehicular Technol., 40: 721-730.

17. Sklar, B., 1997. Rayleigh fading channels in mobile digital communication systems: I. Characterization. IEEE Commun. Magazine, 35: 90-100.

18. Cornelis, C., P. De Kesel, E.E. Kerre, 2004. Shortest paths in fuzzy weighted graphs. Int. J. Intel. Syst., 19: 1051-1068.

19. Yao J.S. and F.T. Lin, 2003. Fuzzy shortest-path network problems with uncertain edge weights. J. Inform. Sci. Eng., 19: 329-351.

20. Zhang, Q. and D.P. Agrawal, 2005. Dynamic probabilistic broadcasting in MANETs. J. Parallel Distributed Comput., 65: 220-233.

21. Tavli, B. and W.B. Heinzelman, 2006. Energy and spatial reuse efficient network-wide real-time data broadcasting in mobile ad hoc networks. IEEE Trans. Vehicular Technol., 5 : 1297-1312. 